OPEN ACCESS

Edited by:

Chuck T. Chen,

National Institute on Alcohol Abuse and Alcoholism (NIAAA),

United States

Reviewed by:

Michinari Nakamura,

Rutgers University-Newark,

United States

Mika Venojärvi,

University of Eastern Finland, Finland

*Correspondence:

Jeongkyu Kim

kimj@cau.ac.kr

Seung-Jin Kim

sjk@kangwon.ac.kr

${ }^{\dagger}$ These authors have contributed equally to this work

Specialty section:

This article was submitted to Clinical and Translational Physiology, a section of the journal Frontiers in Physiology

Received: 07 April 2021 Accepted: 22 June 2021

Published: 16 July 2021

Citation:

Kim HG, Cho J-h, Kim J and Kim S-J (2021) The Role of Epigenetic

Changes in the Progression

of Alcoholic Steatohepatitis.

Front. Physiol. 12:691738.

doi: 10.3389/fphys.2021.691738

\section{The Role of Epigenetic Changes in the Progression of Alcoholic Steatohepatitis}

\author{
Hyeong Geug Kim ${ }^{1}$, Jung-hyo Cho², Jeongkyu Kim ${ }^{3 *+}$ and Seung-Jin Kim ${ }^{4 * t}$ \\ ${ }^{1}$ Department of Biochemistry and Molecular Biology, Indiana University School of Medicine, Indianapolis, IN, United States, \\ ${ }^{2}$ Department of East \& West Cancer Center, Daejeon Korean Medicine Hospital of Daejeon University, Daejeon, \\ South Korea, ${ }^{3}$ Department of Life Science, Chung-Ang University, Seoul, South Korea, ${ }^{4}$ Kangwon Institute of Inclusive \\ Technology, Kangwon National University, Chuncheon, South Korea
}

Alcoholic steatohepatitis (ASH) is a progression hepatitis with severe fatty liver and its mortality rate for 30 -days in patients are over $30 \%$. Additionally, ASH is well known for one-fifth all alcoholic related liver diseases in the world. Excessive chronic alcohol consumption is one of the most common causes of the progression of $\mathrm{ASH}$ and is associated with poor prognosis and liver failure. Alcohol abuse dysregulates the lipid homeostasis and causes oxidative stress and inflammation in the liver. Consequently, metabolic pathways stimulating hepatic accumulation of excessive lipid droplets are induced. Recently, many studies have indicated a link between ASH and epigenetic changes, showing differential expression of alcohol-induced epigenetic genes in the liver. However, the specific mechanisms underlying the pathogenesis of ASH remain elusive. Thus, we here summarize the current knowledge about the roles of epigenetics in lipogenesis, inflammation, and apoptosis in the context of ASH pathophysiology. Especially, we highlight the latest findings on the roles of Sirtuins, a conserved family of class-III histone deacetylases, in ASH. Additionally, we discuss the involvement of DNA methylation, histone modifications, and miRNAs in ASH as well as the ongoing efforts for the clinical translation of the findings in ASH-related epigenetic changes.

Keywords: alcoholic steatohepatitis, epigenetics, sirtuins, DNA methylation, miRNAs

\section{INTRODUCTION}

Alcohol consumption is the leading cause of alcoholic liver diseases (ALDs) worldwide, and it is generally accepted that obesity exacerbate its progression (Parker et al., 2018, 2019; Hwang et al., 2020). ALDs cover a wide range of diseases with various pathological spectrums including simple steatosis, alcoholic steatohepatitis (ASH), progressive liver fibrosis, alcoholic cirrhosis, and

\footnotetext{
Abbreviations: ALD, alcoholic liver disease; ASH, alcoholic steatohepatitis; CXCL1, chemokine (C-X-C motif) ligand 1; DNMT1, DNA methyltransferase 1; GDH, glutamate dehydrogenase; HAT, histone acetyltransferases; HCC, hepatocellular carcinoma; HDACs, histone deacetylases; HDMs, histone demethylases; HFD, high-fat diet; HMTs, histone methyltransferases; LPS, lipopolysaccharide; MAPK, mitogen-activated protein kinase; miRNA, microRNA; MT, metallothionein; NASH, non-alcoholic steatohepatitis; ncRNAs, non-coding RNAs; NF-кB, nuclear factor кB; PPAR $\gamma$, peroxisome proliferator-activated receptor gamma; SAM, S-adenosyl-L-methionine; SIRT, sirtuin; SUMO, small ubiquitinlike modifier; TFs, transcription factors; TNF- $\alpha$, tumor necrosis factor- $\alpha$; $5 \mathrm{hmC}$, 5 -hydroxymethylcytosine; $5 \mathrm{mC}$, 5 Methylcytosine.
} 
hepatocellular carcinoma (HCC) (European Association for the Study of Liver, 2012; Abenavoli et al., 2016). ASH corresponds to the intermediate stage between simple steatosis and progressive liver fibrosis, and its pathophysiological features are mainly characterized by inflammation along with lipid accumulation in the liver (Parker et al., 2018, 2019; Teschke, 2018). In humans, genome-wide association studies conducted in multiple groups have shown that some genetic modifiers, such as PNPLA3, TM6SF2, and MBOAT7 appear to play a pivotal role in ALD progression, however, there are limited studies and data supporting this association in ASH patient samples (Boccuto and Abenavoli, 2017; Meroni et al., 2018). To comprehend the relationship between alcohol consumption and obesity in vivo, Dr. Bin Gao's group in NIAAA developed a rodent model of ASH though the high-fat diet (HFD)plus-binge ethanol challenge, which mimics the concurrent chronic over-nutrition and excessive alcohol consumption in humans. Consequently, they found that over-nutrition and alcohol-drinking synergistically upregulate hepatic and serum Chemokine ( $C-X-C$ motif) ligand 1 (CXCL1) levels (Chang et al., 2015; Hwang et al., 2020). Interestingly, an earlier study had reported that chronic upregulation of chemokines such as CXCL2 is mediated through epigenetic mechanisms, consisting of DNA methylation and histone modification (Kiguchi et al., 2014). Collectively, these observations indicate that over-nutrition and alcohol-drinking synergistically promote ASH through induction of epigenetic genes. Accordingly, in this review we address the roles of epigenetic changes in $\mathrm{ASH}$, whereby the mechanisms underlying ASH progression may be better appreciated to identify a therapeutic target.

\section{ALCOHOLIC STEATOHEPATITIS (ASH)}

In general, ALD is a progressive pathological status generally starting with alcoholic fatty liver disease and progressing to $\mathrm{ASH}$, which is accompanied with inflammation and liver injury. Chronic ASH can lead to liver fibrosis and cirrhosis, and finally to HCC (Seitz et al., 2018; Gao et al., 2019). Among the heavy alcoholic populations, approximately $20 \%$ of them are undergoing ASH condition by evidence of liver biopsy (Stickel and Seitz, 2010); however, no therapeutic currently exists for the treatment of $\mathrm{ASH}$, and liver transplantation is the only treatment strategy in the clinic (Teschke, 2018). Although the exact definition of heavy alcohol users according to each institute, NIAAA normally defines it gender specific for men, more than four drink daily or 14 drinks weekly and three drinks daily or seven drinks weekly for women. On the other hand, SAMHSA mentions people who drinks binge on 5 or more days during last a month ${ }^{1}$. Generally, the etiology of ASH is thought to involve various causes, including genetic, epigenetic, and non(epi)genetic factors, which considerably result in the interindividual variation in ALD phenotype (Anstee et al., 2016). The efforts to elucidate the pathological mechanisms of ASH during

${ }^{1}$ https://www.niaaa.nih.gov/alcohol-health/overview-alcohol-consumption/ moderate-binge-drinking the last decades (Ishak et al., 1991; Jmelnitzky, 1995; Stickel and Seitz, 2010; Rehm et al., 2013; Osna et al., 2017; Teschke, 2018) have shown that the associated ALD is mainly featured by hepatic steatosis, oxidative stress, toxicity due to the production of acetaldehyde, upregulation of inflammatory cytokines, and activation of the chemokine-induced inflammation deriving from the innate immune response (Ishak et al., 1991; Gao and Bataller, 2011; Dunn and Shah, 2016; Osna et al., 2017). Linking these findings may help elucidate the entire pathological steps of $\mathrm{ASH}$, whereby relevant therapeutic approaches can be identified. Thus, we here aim to identify the etiological factors of ASH, especially focusing on epigenetic regulators, which can modulate pathophysiological pathways.

\section{Sirtuins}

Sirtuins (Sirts) are a family of seven silent information regulator 2 proteins (Sirt 1-7), which are a group of Class III histone or protein deacetylases via regulation of nicotinamide adenine dinucleotide $\left(\mathrm{NAD}^{+}\right)$-dependent mode. Sirts mainly regulate cellular protein functions via various posttranslational modifications in differently located subcellular levels. Studies have reported that each type of sirtuin has a different subcellular localization pattern, thus sirtuin family members are critical epigenetic regulators to control energy metabolism, glucose transport and metabolism, and lipid regulations in the liver tissue under the NASH and ASH conditions (Dong et al., 2021). Sirt1 is localized in the nucleus or cytoplasm, being associated with metabolic disorders and inflammation relying on deacetylase activities (Yeung et al., 2004; Lawrence, 2009; Franceschi and Campisi, 2014). The $\mathrm{NAD}^{+}$-dependent deacetylase, Sirt 2 is a cytoplasmic enzyme and mainly takes part in the cell cycle and tumorigenesis (Dryden et al., 2003; Vaquero et al., 2006; North and Verdin, 2007; Wang et al., 2020). Sirt3 is a soluble protein with $\mathrm{NAD}^{+}$-dependent deacetylase activity and exists in the mitochondria. It can regulate metabolic processes, especially the redox homeostasis (Zhang J. et al., 2020). Along with Sirt3, Sirt4 is also observed in the mitochondria and functions as a mitochondrial ADP-ribosyltransferase. Sirt4 suppresses insulin secretion by inhibiting mitochondrial glutamate dehydrogenase (GDH)-1 (Min et al., 2018; Liu et al., 2019). Sirt5 is also located in the mitochondria and shows deacetylase, desuccinylase, and demalonylase activities, which remove acetyl, succinyl, and malonyl groups from the lysine residues of proteins, respectively. Therefore, Sirt5 may regulate the urea cycle, which is involved in the deacetylation of cytochrome $c$, and energy metabolism (Polletta et al., 2015; Sun et al., 2016; Zhang Y. et al., 2017; Chiba et al., 2019). Sirt6 located in the nucleus is a chromatinassociated protein. It is implicated in the modulation of the metabolism, DNA repair, and inflammation (Geng et al., 2020; Klein et al., 2020; Onn et al., 2020). The last member of sirtuins, Sirt7, is localized in the nucleus and its mono-ADPribosyltransferase activity regulates the functions of intracellular regulatory proteins. This sirtuin can be required for ribosomalDNA transcription (Tsai et al., 2012; Mohrin et al., 2015). Contrary to the actively researches of NASH in liver specific roles, ASH studies are rarely reported till recently. This may be attributed by the similarities of phenotypes between NASH and 
ASH, and most of patients did not mention about ALD; however, the specific roles of sirtuins on NASH and ASH are relatively discerned according to the specific molecular targets.

\section{Sirtuins and ASH}

Although an exact role of sirtuins in ALD remains unclearly, previous studies suggested their roles on the inflammation, oxidative stress, and lipid metabolism, respectively. Hepatocyte specific abrogation of Sirtl aggravates hepatic inflammation and steatosis during ALD especially owing to lipid metabolism dysregulation, modification of lipin-1 function (Yin et al., 2014). Sirt 1 also leads to alter SREBP-1c by decrease of hyperacetylation levels (You et al., 2008). Besides, it exerts to reduce H3K9Ac by targeting to the lysine residues in nuclear factor- $\mathrm{\kappa B}$ (NF- $\mathrm{\kappa B}$ ) (Purushotham et al., 2009; Shimazu et al., 2010). Additionally, Sirt1 hepatic deletion mice showed severe inflammatory reactions by Kupffer activation through Sirt1 failed to repress NFATc4 activities (Yin et al., 2014). In NASH, Sirtl not only inactivates KCs cell inactivation, but also leads to lipolysis by increase of $\beta$ oxidation (Vilà et al., 2014). It also inactivates SREBP-1, SCD1 levels, and LKB1, but activates AMPK levels (Cheng et al., 2017).

Contrary to the beneficial effects of Sirt1, hepatic Sirt3 is known to aggravate ALD status during chronic ethanol fed mice model by up-regulation of Sirt3 expression levels. Especially, liver specific Sirt3 knocking-down mice led to ameliorations of ASH condition by improvement of autophagy, especially increases of LC3B II in hepatic protein levels. Accordance with the above outcomes, Sirt3 mediated autophagy induction condition of ethanol treated hepatocyte cells, AML 12 cells, showed cytoprotective effects on ethanol treated condition by inhibition of pro-apoptotic proteins Bax, but activation of $\mathrm{Bcl} 2$, respectively (Ma et al., 2019). During NASH by MCD diet fed mice model, hepatic Sirt3 is involved lipotoxicity mediated apoptosis by acetylation and mitochondrial respiratory complexes III and IV inactivation and regulates SOD2 activities (Kendrick et al., 2011; He et al., 2013). The roles of Sirt5 in ALD, previous study reported that hepatic protein levels of Sirt5 were depleted by 4 weeks of alcohol diet of wild type SD rat model. This condition allowed to communication between mitochondria and nucleus levels by evidences of hypophosphorylation of FoxO1, hyperacetylation of p53, and mitochondrial PGC-1a, respectively (Ma et al., 2019).

Hepatic tissue specific deletion of Sirt6 in mice which was applied Mx1-Cre (for all liver cell types KO conditions) causes severe ALD by increasing hepatic oxidation and inflammation, which are regulated by hepatic metallothionein 1 and 2 (Mt1 and Mt2). Conversely, over expression of Sirt6 in the liver prevents the above-mentioned pathological alterations by enhancing cellular antioxidant activities and anti-inflammatory reactions, accompanied by upregulation of MT1/2. Sirt6 not only synergistically increases both Mt1 and Mt2 levels during the physiological status but also modulates hepatic MTs through controlling MTF1-induced acetylation (Kim et al., 2019; Figure 1A). On the other hand, liver tissue specific $\mathrm{KO}$ condition of Sirt6 activates deacetylation of Smad3 at lysine residues K333 and K378 in HSCs, during liver fibrosis development (Zhong et al., 2020).

\section{DNA METHYLATION}

DNA methylation is well documented in the progression of ASH due to its specific role of epigenetic regulator to modulate transcription factors (TFs) as well as chromatin accessibility. DNA methylation, such as 5-Methylcytosine (5mC) and 5-hydroxymethylcytosine $(5 \mathrm{hmC})$, which are located near promoters with no or low transcriptional activity (Bird, 2002). DNA methylation has been uncovered to serve as the molecular basis of ALD (Zeybel et al., 2015).

Upon the function of the DNA methylation during ALD, it causes an alteration in the methionine metabolism via changes in the expression of various genes; however, the role of epigenetic machinery still remains unclearly. As major epigenetic target molecules of DNA modification both $5 \mathrm{mC}$ and $5 \mathrm{hmC}$ lead to modification of gene expression by regulating of TFs, mainly suppress certain genes transcription levels (Gopalakrishnan et al., 2008; Argentieri et al., 2017).

DNA methylation-related epigenetic regulation in the biopsy samples from ASH patients were severely modified by DNA methyltransferase 1 (DNMT1) and DNMT3B through increasing genomewide $5 \mathrm{mC}$ methylation level (Liu et al., 2014). One of considerable mechanism in linking DNA methylation and ASH is UFMylation, a biological process that attaches ubiquitously expressed small ubiquitin fold modifier 1 (UFM1), the most recently identified ubiquitin-like molecule, to target proteins (Wei and Xu, 2016). Although its particular role in ALD is not clearly revealed, the Ufmylation levels were lowered in ASH and this modification was led by increases in DNA methylation levels on promoter CpG of Ufm1, Ufc1, and UfSP1, which are correlated with increased DNMT1 and DNMT3B mRNA levels in AH patients (Liu et al., 2014; Shen et al., 2015).

Plasma DNA methylation at the gene promoter of PPAR $\gamma$ was increased in ALD-provoked liver cirrhosis patients by hypermethylation manners and increased at the site of particular loci in PDGF $\alpha$ gene. Interestingly the above methylation levels of $\operatorname{PPAR} \gamma$ were higher in hepatocyte than myofibroblast in hepatic fibrosis patients of ALD (Hardy et al., 2017). Regarding lipid homeostasis and fibrosis related genes including PPAR $\alpha, \operatorname{PPAR} \alpha$, and TGF $\beta 1$, Collagen $1 \mathrm{~A} 1$, PDGF $\alpha$ genes in ALD patients, contrary to NAFLD patients show different patterns especially increase of DNA methylation at the site of TGF $\beta 1$ exon was upregulated in CpG2, but reverse manner of Collagen 1A1 gene (Zeybel et al., 2015; Figure 1B).

\section{HISTONE MODIFICATIONS}

In addition to DNA methylation, histone modifications including histone methylation, acetylation, phosphorylation, and ubiquitination, etc., are also well known as a key epigenetic regulator. It is reported that several key metabolites are involved in each histone modification such as acetate, S-adenosylmethionine (SAM), $\mathrm{NAD}^{+}$, and zinc are relevant to alcohol metabolism, especially in ASH (Moghe et al., 2011). Herein, thus, we next focus on methylation, acetylation, phosphorylation, ubiquitination, and sumoylation of histone 

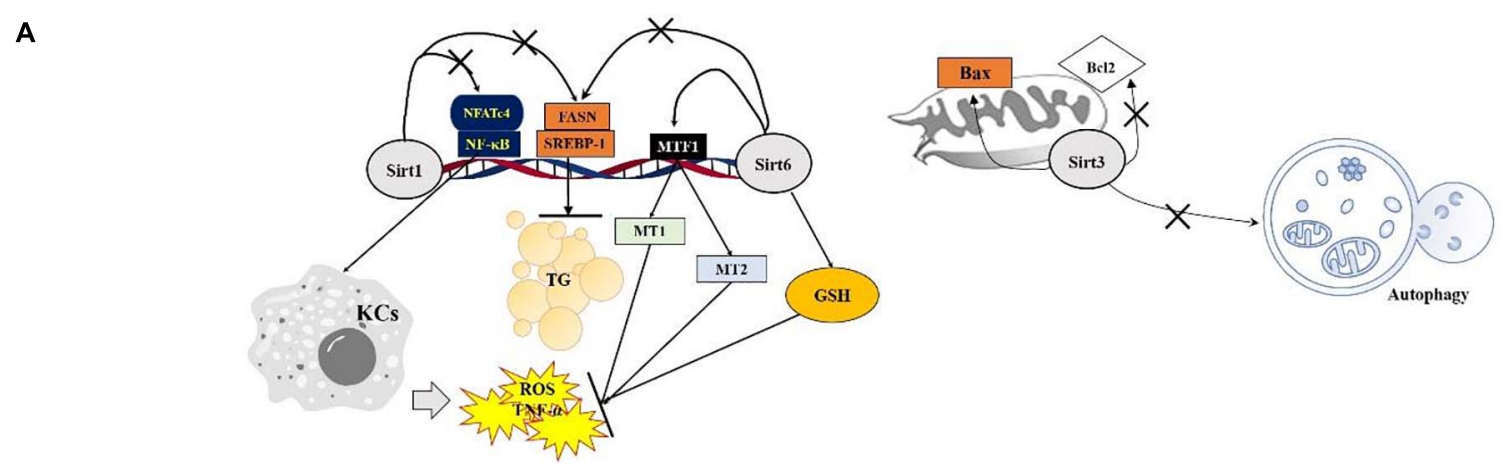

B
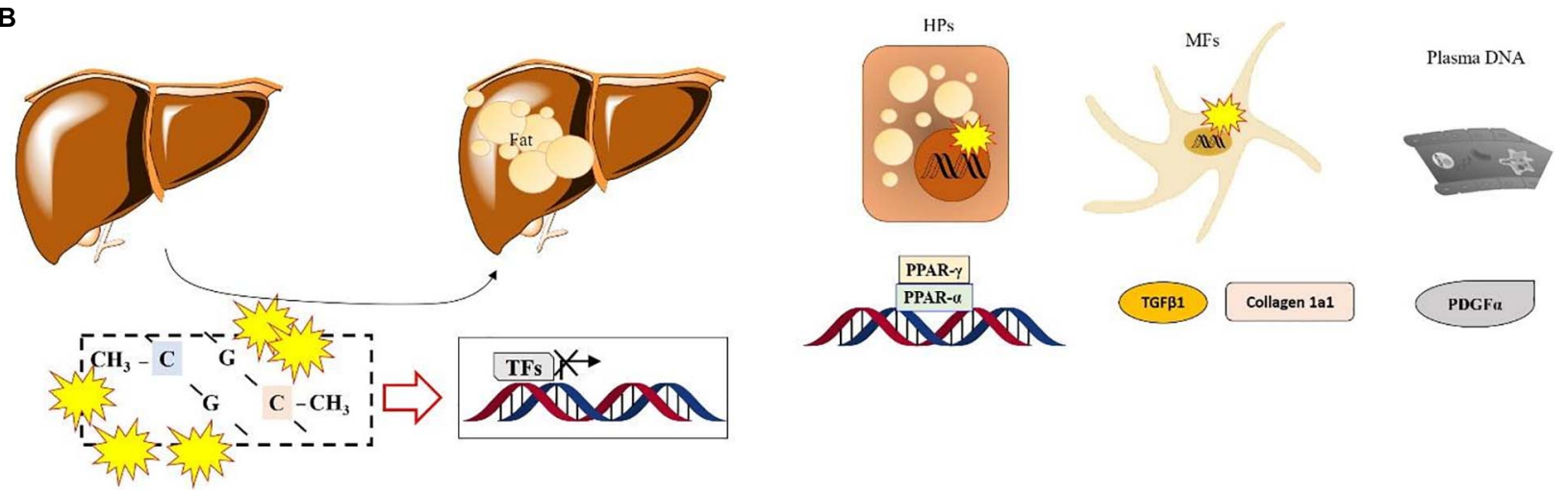

FIGURE 1 | Specific roles of Sirtuins and DNA methylation in ASH. (A) Numerous SIRT-modulated mechanisms mainly regulate hepatic inflammation via inactivation of Kupffer cells and reduce lipogenesis during ASH. Sirt6 shows protective effects by normalizing the ethanol metabolism and increasing antioxidant components via enhancement of MTF1 acylation in the nucleus. Sirt3 enhances the mitochondrial apoptotic signals and prevents autophagy. (B) The role of DNA methylation in ASH. Lipid-droplet accumulation in the liver during ASH is suppressed by regulating factors associated with the lipid homeostasis or extracellular matrix via the Ufmylation pathways in hepatocytes and myofibroblasts. In plasma DNA, DNA methylation also regulates PDGF $\alpha$ expression during ASH.

during ASH development via emphasis on their specific roles of epigenetic modulations, especially gene expression (Table 1 ).

\section{Histone Methylation}

Histone methylation is a dynamic process with important roles in development and differentiation (Kouzarides, 2007; Eissenberg and Shilatifard, 2010), which are being catalyzed by two groups of enzymes, histone methyltransferases (HMTs) and histone demethylases (HDMs), respectively. First, HMTs catalyze the transfer of methyl group(s) from the cofactor S-adenosyl-Lmethionine (SAM), the primary methyl group donor, most heavily to lysine or arginine residue of histone $\mathrm{H} 3$ followed by that of histone $\mathrm{H} 4$, affecting in turn the recruitment and binding of several regulatory proteins to chromatin (Morera et al., 2016; Hyun et al., 2017; Kaniskan et al., 2018). According to the accumulated evidence revealed that ASH is deeply linked to decreased level of SAM in the liver tissues from rodents and non-human primates (Lieber, 2002; King et al., 2016), which suggest that the transfer of methyl group catalyzed by HMTs can be interrupted and ultimately affects global pattern of gene expression. Additionally, another study performed in rodents also reported that acute and chronic ethanol exposures altered the expression levels of several susceptible genes which are associated with site specific histone methylation at their regulatory regions by performance of both in vitro and in vivo experiments (PalBhadra et al., 2007; Mandrekar, 2011). It should also be noted that ethanol consumption generally induced deleterious effect on the inflammatory response because it is well accepted that upon ethanol exposure one of a key pro-inflammatory cytokine, tumor necrosis factor- $\alpha$ (TNF- $\alpha)$ is silenced by H3K9 methylation (Nagy, 2015). Together, it is evident that mapping the pattern of histone methylation in response to alcohol exposure would help us better understanding the underlying mechanisms of ASH progression.

\section{Histone Acetylation}

Global changes in histone modifications upon ethanol exposure suggest that they would be resulted in genomewide alterations in gene expression; however, some cases for depending on types of alcohol exposures histone acetylation, is modulated by two types of specific enzymes such as histone acetyltransferases (HATs) and histone deacetylases (HDACs), can be limited to a type of subsets of genes. Indeed, an increased gene-selective levels of histone $\mathrm{H} 3 \mathrm{~K} 9$ 
TABLE 1 | Summary of histone modifications and miRNAs involved in ASH.

\begin{tabular}{|c|c|c|c|c|}
\hline $\begin{array}{l}\text { Type of epigenetic } \\
\text { modification }\end{array}$ & $\begin{array}{l}\text { Alteration upon } \\
\text { exposure to } \\
\text { alcohol }\end{array}$ & $\begin{array}{l}\text { Targets altered by } \\
\text { epigenetic changes }\end{array}$ & Effect upon exposure to alcohol & References \\
\hline & $\mathrm{H} 3 \mathrm{~K} 4 \mathrm{me} \uparrow$ & Adh $\uparrow$, GST-yc $2 \uparrow$ & $\begin{array}{l}\text { Speed up ethanol metabolism; Progression } \\
\text { of fatty liver, Inflammation and liver cirrhosis }\end{array}$ & Pal-Bhadra et al., 2007 \\
\hline \multirow[t]{2}{*}{ Histone methylation } & $\mathrm{H} 3 \mathrm{~K} 9 \mathrm{me} \downarrow$ & Lsdh $\downarrow$, cytP4502c11 $\downarrow$ & $\begin{array}{l}\text { Progression of fatty liver, Inflammation and } \\
\text { liver cirrhosis }\end{array}$ & Pal-Bhadra et al., 2007 \\
\hline & $\mathrm{H} 3 \mathrm{~K} 9 \mathrm{me} \uparrow$ & $\mathrm{TNF}-\alpha \downarrow$ & Exacerbation of the inflammatory response & Nagy, 2015 \\
\hline Histone acetylation & H3K9ac $\uparrow$ & TNF- $\alpha \uparrow ; P N P L A 3 \uparrow$ & $\begin{array}{l}\text { HAT activity and HDAC inhibition; } \\
\text { exacerbation of the inflammatory response }\end{array}$ & Nagy, 2015; Restrepo et al., 2017 \\
\hline Histone phosphorylation & 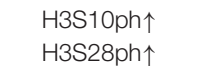 & 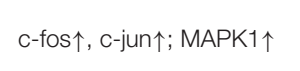 & Transcriptional activation & $\begin{array}{l}\text { Lee and Shukla, 2007; James et al., } \\
2012\end{array}$ \\
\hline \multirow{3}{*}{ miRNAs } & $\begin{array}{l}\operatorname{miR}-155 \uparrow \\
\operatorname{miR}-21 \uparrow\end{array}$ & TNF- $\alpha \uparrow, I L-1 \beta \uparrow$, PPAR $\gamma \downarrow$ & $\begin{array}{l}\text { Increase inflammation response and } \\
\text { Steatosis }\end{array}$ & $\begin{array}{l}\text { Hartmann and Tacke, 2016; Zhang T. } \\
\text { et al., } 2020\end{array}$ \\
\hline & $\operatorname{miR}-212 \uparrow$ & $\mathrm{ZO}-1 \downarrow$ & Increased gut and liver injury & Tang et al., 2015 \\
\hline & $\operatorname{miR}-122 \uparrow \downarrow$ & HIF-1 $\alpha \uparrow ; P G C 1 a \uparrow ; A p o E \uparrow$ & $\begin{array}{l}\text { Lipid synthesis, export and cholesterol } \\
\text { homeostasis }\end{array}$ & $\begin{array}{l}\text { Krützfeldt et al., 2005; Esau et al., } \\
\text { 2006; Bala et al., 2012; McDaniel et al., } \\
\text { 2014; Momen-Heravi et al., } 2015\end{array}$ \\
\hline
\end{tabular}

acetylation in the liver tissue is observed by chronic ethanol feeding using rat model without global changes in histone acetylation at other lysine residues such as $\mathrm{H} 3 \mathrm{~K} 14, \mathrm{H} 3 \mathrm{~K} 18$, and H3K23, respectively (Park et al., 2003, 2012), pointing to an existence of disease relevant epigenetic signatures during the progression of ASH.

In addition, it is now generally accepted that in the liver, level of histone $\mathrm{H} 3$ acetylation and alcohol induced fatty liver depend on the balance between the activity of HATs and HDACs being affected by alcohol consumption in rat hepatocytes and patients with Rheumatoid arthritis and osteoarthritis (Ito and Adcock, 2002; Park et al., 2005; Huber et al., 2007). For example, ethanolregulated HAT and GCN5 in human cell lines modulates the expression of PGC1b, a protein involving in fat metabolism (Kelly et al., 2009; Choudhury et al., 2011). Intriguingly, ASH condition of rat model leads to increases of another HAT called p300 levels in the cellular nuclei at a peak blood alcohol level, which is correlated with increased H3K9 acetylation activities (BardagGorce et al., 2007). In the case of HDACs, aforementioned SIRT1 plays a pivotal role in the pathogenesis of ASH via impairments of SIRT1 and SIRT1-regulated genes encoding lipogenic or fatty acid oxidation enzymes (Yin et al., 2012). Accumulating evidences recommended that both HATs and HDACs are likely to play a role in ethanol-induced liver injury. This implies again that a certain type of features shown in histone acetylation can be used as biomarker of a certain disease such as ASH (Park et al., 2005; Shepard et al., 2008; Kirpich et al., 2012; Pochareddy and Edenberg, 2012).

\section{Histone Phosphorylation}

Contrary to histone methylation and acetylation, histone phosphorylation is mediated by two types of enzymes including kinases and phosphates, having opposing modes of action, which works together with other histone modifications, thus generating the platform for mutual interactions between the modifications under the ASH condition. Under the acute ethanol exposure status, histone $\mathrm{H} 3 \mathrm{~S} 10$ and $\mathrm{H} 3 \mathrm{~S} 28$ phosphorylation which were depended on p38 mitogenactivated protein kinase (MAPK), are directly able to affect acetylation levels at two amino acid residues of the same histone $\mathrm{H} 3 \mathrm{~K} 9$ and $\mathrm{H} 3 \mathrm{~K} 14$ acetylation (Lo et al., 2000; Edmondson et al., 2002; Lee and Shukla, 2007; Aroor et al., 2010). Furthermore, H3S10 phosphorylation can induce transactivation by interaction with H4K16Ac, suggesting a synergistic relationship between phosphorylation and acetylation in transcription regulation on a subset of genes in response to alcohol exposure. For example, both histone H3S10 phosphorylation and the same histone H3K14 acetylation are known to be involved in cytokine-induced gene expression mediated by nuclear IKK $\alpha$ leading to NF- $\kappa \mathrm{B}$ activation (Anest et al., 2003; Yamamoto et al., 2003). Intriguingly, retinoic acid receptor- $\beta$ and $c$-jun gene regulation is not linked to histone $\mathrm{H} 3$ acetylation but histone $\mathrm{H} 3$ phosphorylation (Clayton et al., 2000; Thomson et al., 2001; Lefebvre et al., 2002) indicating that these two epigenetic changes can occur independently. Great effort is therefore needed to understand whether alcohol induces crosstalk between for instance histone phosphorylation and acetylation or as independent pathways to regulate target gene expression in $\mathrm{ASH}$, because the various histone modifications observed in cultured hepatocytes in response to ethanol follow different time courses.

\section{Other Histone Modifications}

Unlike other histone modifications as mentioned above, histone ubiquitination occurs in a variety of histones, including histone $\mathrm{H} 1, \mathrm{H} 2 \mathrm{~A}, \mathrm{H} 2 \mathrm{~B}$, and $\mathrm{H} 3$. Especially, mono-ubiquitination has a critical role in the translocation of proteins, DNAdamage signaling, and transcriptional regulation. $\mathrm{H} 2 \mathrm{~A}$ mono-ubiquitination is generally associated with gene 


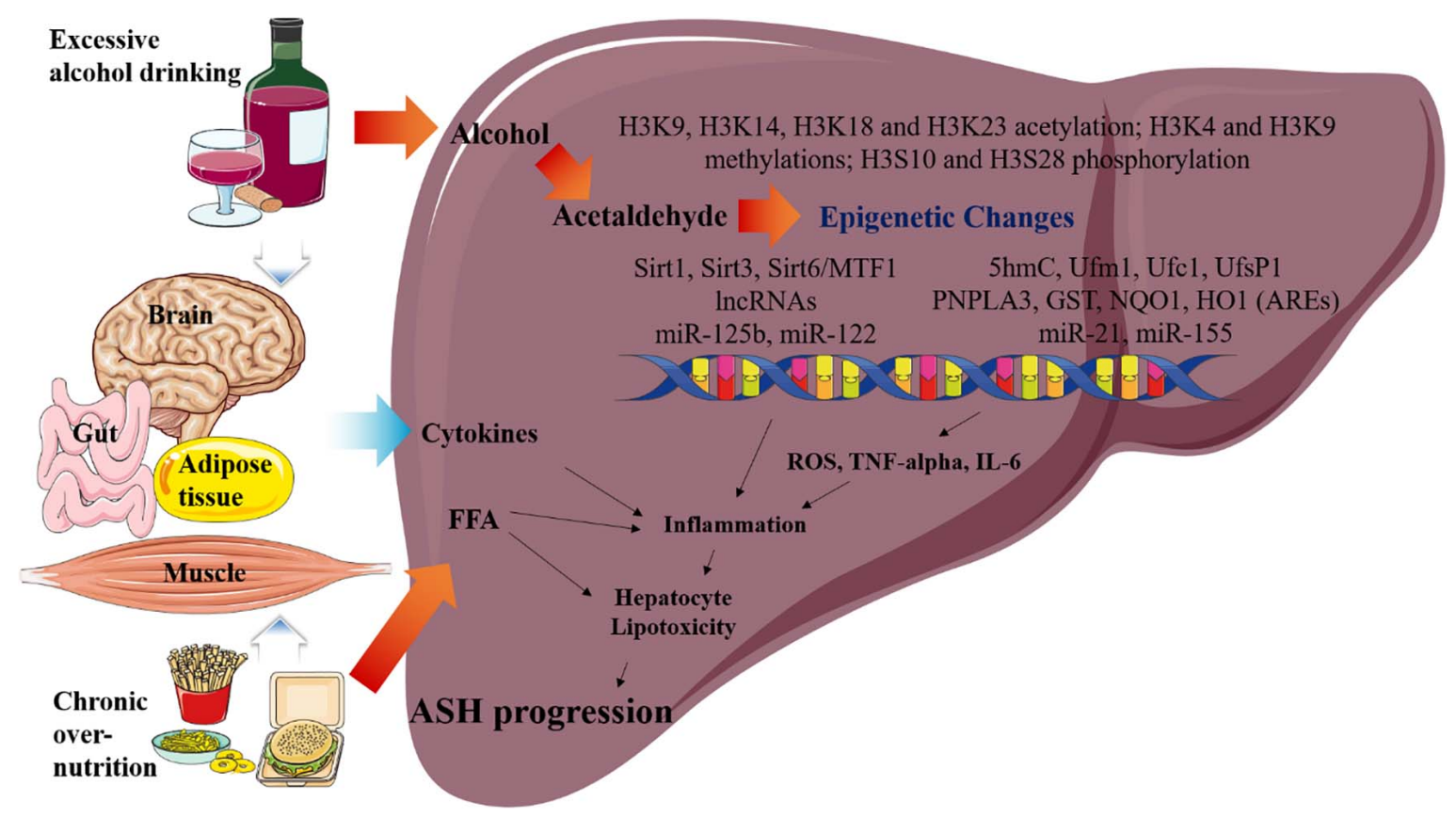

FIGURE 2 | Overview of the epigenetic changes in ASH. Metabolic pathways regulated by acetaldehyde, cytokine, and FFA are induced by excessive alcohol drinking and chronic over- nutrition. Excessive alcohol drinking and chronic over-nutrition synergistically induces ASH progression via activating several different signaling pathways in the liver, including epigenetic changes. Additionally, hepatic cytokine and FFA levels of cytokines are induced as a result of the associated multiple organ damages, such as those in the adipose tissue, brain, intestines, and muscles. Eventually, this pathway increases hepatic inflammation and hepatocyte lipotoxicity, which result in the stimulation of $\mathrm{ASH}$.

silencing, whereas $\mathrm{H} 2 \mathrm{~B}$ mono-ubiquitination is correlated with transcriptional activation (Zhang, 2003). Similar to histone phosphorylation, histone mono-ubiquitination is known to induce acetylation of the same histone (Zhang $\mathrm{X}$. et al., 2017). The effects of alcohol consumption on the histone ubiquitination in the liver have not been studied in detail. However, a previous study of transcriptional profiling of post-mortem brain samples from individuals with a history of alcohol abuse or dependence showed abnormalities in the ubiquitin signaling (Ishino et al., 2014). Accordingly, it could be legitimate to presume that alcohol exposure may exacerbate histone ubiquitination.

Histone sumoylation is another post-translational modification involved in diverse biological processes, including the stress response, protein stability, the cell cycle, apoptosis, nucleocytoplasmic transport, and transcriptional regulation. Histone sumoylation occurs on lysine residues and is known to be a transcriptionally repressive modification (Rubio et al., 2013). Small ubiquitin-like modifier (SUMO)-conjugated yeast histones appear to suppress gene expression by opposing active histone marks, such as acetylation or ubiquitylation (Nathan et al., 2006). Although only a few studies have explored how alcohol consumption affect histone sumoylation, a study has mentioned that the sumoylation in the livers of mice fed with ethanol is significantly reduced relative to the levels in the control mice (Nisticò et al., 2014). This finding implies alcohol-mediated changes in the level of histone sumoylation. Together, much more attention should be given to those modifications to better understand the complexity of epigenetic networks involved in the pathogenesis of ASH.

\section{MICRORNAs IN ASH}

In addition to histone modifications, miRNAs, regulator of post-transcriptional gene expression, are abundant in the liver and play key roles in diverse biological processes associated with liver injury, including hepatocyte regeneration, apoptosis, and inflammation (Schueller et al., 2018). It should be noted that the levels of a subset of miRNAs was found to be dysregulated upon alcohol consumption. Deregulation of the levels of some specific miRNAs has commonly been identified, suggesting the importance of these factors in alcoholic liver injury. Numerous reports on miRNA dysregulation in alcoholinduced liver diseases summarize the up- and down-regulated miRNAs in the liver in response to alcohol consumption (Table 1), and these differentially expressed miRNAs may act as causative factors of ASH. Although changes in miRNA levels can affect the expression of the enzymes involved in other epigenetic modifications, expression of miRNAs themselves can be subject to regulation via histone modifications that regulate miRNA expression. For example, ethanol upregulates miR-155 via the recruitment of a transcriptional activator called NF- $\kappa B$ to the miR-155 promoter (McDaniel et al., 2014), subsequently causing epigenetic changes associated with gene activation. MiR155 is also known as a regulator of the hepatic secretion of 
inflammatory agents, such as lipopolysaccharide (LPS)-induced TNF- $\alpha$, consistent with a pathogenic role in the liver in response to acute or chronic alcohol consumption (Bala et al., 2011). More recently, growing evidence suggests that circulating miRNAs can be used as stable biomarkers for alcohol-induced liver diseases because similar miRNA expression patterns are found both in the liver and blood of ALD patients. Moreover, miRNAs can also stay in the plasma in a stable form as well as in the blood of ALD patients, making these small RNAs feasible biomarkers for the detection of ALD and other liver disorders, suggesting that the effect of acute or chronic alcohol consumption on the liver may be defined by quantifying circulating miRNAs taken from serum or plasma. It has been therefore increasingly appreciated that understanding the role of miRNAs in ASH has many potential aspects that may be applied into novel therapeutic approaches in ASH. Although there is currently no miRNA-related treatment modality in ASH, miRNAs may be utilized as key therapeutic targets in ALD in the near future.

\section{CONCLUSION AND FUTURE PERSPECTIVES}

Over the past decade, an increased understanding of the relationship between epigenetic factors and $\mathrm{ASH}$ has been developed. Nevertheless, the specific epigenetic mechanisms underlying the pathophysiology of ASH still remain unclear. Thus, we addressed the important roles of the epigenetic regulators that can modulate the pathophysiological pathways

\section{REFERENCES}

Abenavoli, L., Masarone, M., Federico, A., Rosato, V., Dallio, M., Loguercio, C., et al. (2016). Alcoholic hepatitis: pathogenesis, diagnosis and treatment. Rev. Recent Clin. Trials 11, 159-166. doi: 10.2174/1574887111666160724183409

Anest, V., Hanson, J. L., Cogswell, P. C., Steinbrecher, K. A., Strahl, B. D., and Baldwin, A. S. (2003). A nucleosomal function for ikappab kinase-alpha in nf-kappab-dependent gene expression. Nature 423, 659-663. doi: 10.1038/ nature 01648

Anstee, Q. M., Seth, D., and Day, C. P. (2016). Genetic factors that affect risk of alcoholic and nonalcoholic fatty liver disease. Gastroenterology 150, 1728.e1744.e. doi: 10.1053/j.gastro.2016.01.037

Argentieri, M. A., Nagarajan, S., Seddighzadeh, B., Baccarelli, A. A., and Shields, A. E. (2017). Epigenetic pathways in human disease: the impact of DNA methylation on stress-related pathogenesis and current challenges in biomarker development. EBioMedicine 18, 327-350. doi: 10.1016/j.ebiom.2017.03.044

Aroor, A. R., James, T. T., Jackson, D. E., and Shukla, S. D. (2010). Differential changes in map kinases, histone modifications, and liver injury in rats acutely treated with ethanol. Alcohol Clin. Exp. Res. 34, 1543-1551. doi: 10.1111/j.15300277.2010.01239.x

Bala, S., Marcos, M., Kodys, K., Csak, T., Catalano, D., Mandrekar, P., et al. (2011). Up-regulation of microrna-155 in macrophages contributes to increased tumor necrosis factor \{alpha\} (tnf\{alpha\}) production via increased mrna half-life in alcoholic liver disease. J. Biol. Chem. 286, 1436-1444. doi: 10.1074/jbc.M110. 145870

Bala, S., Petrasek, J., Mundkur, S., Catalano, D., Levin, I., Ward, J., et al. (2012). Circulating microRNAs in exosomes indicate hepatocyte injury and inflammation in alcoholic, drug-induced, and inflammatory liver diseases. Hepatology 56, 1946-1957. doi: 10.1002/hep.25873

Bardag-Gorce, F., French, B. A., Joyce, M., Baires, M., Montgomery, R. O., Li, J., et al. (2007). Histone acetyltransferase p300 modulates gene expression in an in ASH. Particularly our study focuses on the following areas of current research on the epigenetic changes reported in $\mathrm{ASH}$, mainly sirtuins, histone acetylation, methylation, and phosphorylation. These epigenetic changes increase cytokine activation, inflammation, and lipogenesis in the liver, eventually leading to ASH progression (Figure 2). In the future, these epigenetic regulators as well as the ASH-related metabolic pathways may be targeted for the treatment of ASH. Moreover, further investigation of these epigenetic regulators may also lead to effective treatment modalities for metabolic diseases, including ALD.

\section{AUTHOR CONTRIBUTIONS}

HK designed and wrote the manuscript. J-HC critically revised the manuscript. S-JK and JK supervised the whole project and wrote the manuscript. All authors contributed to the article and approved the submitted version.

\section{FUNDING}

This work was supported by the National Research Foundation of Korea (NRF) grant funded by the Korea government (MSIT) (NRF-2020R1F1A1050534, NRF-2020R1A5A8019180, and NRF2020R1F1A1076781) and 2020 Research Grant from Kangwon National University (S-JK) and 2020 Research Grant from Chung-Ang University (JK).

epigenetic manner at high blood alcohol levels. Exp. Mol. Pathol. 82, 197-202. doi: 10.1016/j.yexmp.2006.10.006

Bird, A. (2002). DNA methylation patterns and epigenetic memory. Genes Dev. 16, 6-21. doi: 10.1101/gad.947102

Boccuto, L., and Abenavoli, L. (2017). Genetic and epigenetic profile of patients with alcoholic liver disease. Ann. Hepatol. 16, 490-500. doi: 10.5604/01.3001. 0010.0274

Chang, B., Xu, M. J., Zhou, Z., Cai, Y., Li, M., Wang, W., et al. (2015). Shortor long-term high-fat diet feeding plus acute ethanol binge synergistically induce acute liver injury in mice: an important role for cxcl1. Hepatology 62, 1070-1085. doi: 10.1002/hep.27921

Cheng, J., Liu, C., Hu, K., Greenberg, A., Wu, D., Ausman, L. M., et al. (2017). Ablation of systemic sirt1 activity promotes nonalcoholic fatty liver disease by affecting liver-mesenteric adipose tissue fatty acid mobilization. Biochim. Biophys. Acta Mol. Basis Dis. 1863, 2783-2790. doi: 10.1016/j.bbadis.2017. 08.004

Chiba, T., Peasley, K. D., Cargill, K. R., Maringer, K. V., Bharathi, S. S., Mukherjee, E., et al. (2019). Sirtuin 5 regulates proximal tubule fatty acid oxidation to protect against aki. J. Am. Soc. Nephrol. 30, 2384-2398. doi: 10.1681/asn. 2019020163

Choudhury, M., Pandey, R. S., Clemens, D. L., Davis, J. W., Lim, R. W., and Shukla, S. D. (2011). Knock down of gen5 histone acetyltransferase by sirna decreases ethanol-induced histone acetylation and affects differential expression of genes in human hepatoma cells. Alcohol 45, 311-324. doi: 10.1016/j.alcohol.2010. 12.003

Clayton, A. L., Rose, S., Barratt, M. J., and Mahadevan, L. C. (2000). Phosphoacetylation of histone h3 on c-fos- and c-jun-associated nucleosomes upon gene activation. Embo J. 19, 3714-3726. doi: 10.1093/emboj/19.14.3714

Dong, X. C., Chowdhury, K., Huang, M., and Kim, H. G. (2021). Signal transduction and molecular regulation in fatty liver disease. Antioxid. Redox Signal doi: 10.1089/ars.2021.0076 [Epub ahead of print]. 
Dryden, S. C., Nahhas, F. A., Nowak, J. E., Goustin, A. S., and Tainsky, M. A. (2003). Role for human sirt2 nad-dependent deacetylase activity in control of mitotic exit in the cell cycle. Mol. Cell Biol. 23, 3173-3185. doi: 10.1128/mcb.23.9.31733185.2003

Dunn, W., and Shah, V. H. (2016). Pathogenesis of alcoholic liver disease. Clin. Liver Dis. 20, 445-456. doi: 10.1016/j.cld.2016.02.004

Edmondson, D. G., Davie, J. K., Zhou, J., Mirnikjoo, B., Tatchell, K., and Dent, S. Y. (2002). Site-specific loss of acetylation upon phosphorylation of histone h3. J. Biol. Chem. 277, 29496-29502. doi: 10.1074/jbc.M200651200

Eissenberg, J. C., and Shilatifard, A. (2010). Histone h3 lysine 4 (h3k4) methylation in development and differentiation. Dev. Biol. 339, 240-249. doi: 10.1016/j. ydbio.2009.08.017

Esau, C., Davis, S., Murray, S. F., Yu, X. X., Pandey, S. K., Pear, M., et al. (2006). Mir-122 regulation of lipid metabolism revealed by in vivo antisense targeting. Cell Metab. 3, 87-98. doi: 10.1016/j.cmet.2006.01.005

Franceschi, C., and Campisi, J. (2014). Chronic inflammation (inflammaging) and its potential contribution to age-associated diseases. J. Gerontol. A Biol. Sci. Med. Sci. 69(Suppl. 1), S4-S9. doi: 10.1093/gerona/glu057

Gao, B., and Bataller, R. (2011). Alcoholic liver disease: pathogenesis and new therapeutic targets. Gastroenterology 141, 1572-1585. doi: 10.1053/j.gastro. 2011.09.002

Gao, B., Ahmad, M. F., Nagy, L. E., and Tsukamoto, H. (2019). Inflammatory pathways in alcoholic steatohepatitis. J. Hepatol. 70, 249-259. doi: 10.1016/j. jhep.2018.10.023

Geng, A., Tang, H., Huang, J., Qian, Z., Qin, N., Yao, Y., et al. (2020). The deacetylase sirt6 promotes the repair of uv-induced DNA damage by targeting ddb2. Nucleic Acids Res. 48, 9181-9194. doi: 10.1093/nar/gkaa661

Gopalakrishnan, S., Van Emburgh, B. O., and Robertson, K. D. (2008). DNA methylation in development and human disease. Mutat. Res. 647, 30-38. doi: 10.1016/j.mrfmmm.2008.08.006

Hardy, T., Zeybel, M., Day, C. P., Dipper, C., Masson, S., McPherson, S., et al. (2017). Plasma DNA methylation: a potential biomarker for stratification of liver fibrosis in non-alcoholic fatty liver disease. Gut 66, 1321-1328. doi: 10. 1136/gutjnl-2016-311526

Hartmann, P., and Tacke, F. (2016). Tiny rna with great effects: mir-155 in alcoholic liver disease. J. Hepatol. 64, 1214-1216. doi: 10.1016/j.jhep.2016.02.039

He, J., Hu, B., Shi, X., Weidert, E. R., Lu, P., Xu, M., et al. (2013). Activation of the aryl hydrocarbon receptor sensitizes mice to nonalcoholic steatohepatitis by deactivating mitochondrial sirtuin deacetylase sirt3. Mol. Cell. Biol. 33, 2047-2055. doi: 10.1128/mcb.01658-12

Huber, L. C., Brock, M., Hemmatazad, H., Giger, O. T., Moritz, F., Trenkmann, M., et al. (2007). Histone deacetylase/acetylase activity in total synovial tissue derived from rheumatoid arthritis and osteoarthritis patients. Arthritis Rheum. 56, 1087-1093. doi: 10.1002/art.22512

Hwang, S., Ren, T., and Gao, B. (2020). Obesity and binge alcohol intake are deadly combination to induce steatohepatitis: a model of high-fat diet and binge ethanol intake. Clin. Mol. Hepatol. 26, 586-594. doi: 10.3350/cmh.2020.0100

Hyun, K., Jeon, J., Park, K., and Kim, J. (2017). Writing, erasing and reading histone lysine methylations. Exp. Mol. Med. 49:e324. doi: 10.1038/emm.2017.11

Ishak, K. G., Zimmerman, H. J., and Ray, M. B. (1991). Alcoholic liver disease: pathologic, pathogenetic and clinical aspects. Alcohol Clin. Exp. Res. 15, 45-66. doi: 10.1111/j.1530-0277.1991.tb00518.x

Ishino, Y., Hayashi, Y., Naruse, M., Tomita, K., Sanbo, M., Fuchigami, T., et al. (2014). Brela, a histone h2b ubiquitin ligase, regulates the cell cycle and differentiation of neural precursor cells. J. Neurosci. 34, 3067-3078. doi: 10. 1523/jneurosci.3832-13.2014

Ito, K., and Adcock, I. M. (2002). Histone acetylation and histone deacetylation. Mol. Biotechnol. 20, 99-106. doi: 10.1385/mb:20:1:099

James, T. T., Aroor, A. R., Lim, R. W., and Shukla, S. D. (2012). Histone h3 phosphorylation (ser10, ser28) and phosphoacetylation (k9s10) are differentially associated with gene expression in liver of rats treated in vivo with acute ethanol. J. Pharmacol. Exp. Ther. 340, 237-247. doi: 10.1124/jpet. 111.186775

Jmelnitzky, A. C. (1995). new suggestions for the management of alcoholic liver diseases. Acta Gastroenterol. Latinoam. 25, 73-84.

Kaniskan, H., Martini, M. L., and Jin, J. (2018). Inhibitors of protein methyltransferases and demethylases. Chem. Rev. 118, 989-1068. doi: 10.1021/ acs.chemrev.6b00801
Kelly, T. J., Lerin, C., Haas, W., Gygi, S. P., and Puigserver, P. (2009). Gcn5-mediated transcriptional control of the metabolic coactivator pgc-1beta through lysine acetylation. J. Biol. Chem. 284, 19945-19952. doi: 10.1074/jbc. M109.015164

Kendrick, A. A., Choudhury, M., Rahman, S. M., McCurdy, C. E., Friederich, M., Van Hove, J. L., et al. (2011). Fatty liver is associated with reduced sirt3 activity and mitochondrial protein hyperacetylation. Biochem. J. 433, 505-514. doi: 10.1042/bj20100791

Kiguchi, N., Saika, F., Kobayashi, Y., and Kishioka, S. (2014). Epigenetic regulation of cc-chemokine ligand 2 in nonresolving inflammation. Biomol. Concepts. 5, 265-273. doi: 10.1515/bmc-2014-0022

Kim, H. G., Huang, M., Xin, Y., Zhang, Y., Zhang, X., Wang, G., et al. (2019). The epigenetic regulator sirt6 protects the liver from alcohol-induced tissue injury by reducing oxidative stress in mice. J. Hepatol. 71, 960-969. doi: 10.1016/j.jhep. 2019.06.019

King, A. L., Mantena, S. K., Andringa, K. K., Millender-Swain, T., DunhamSnary, K. J., Oliva, C. R., et al. (2016). The methyl donor s-adenosylmethionine prevents liver hypoxia and dysregulation of mitochondrial bioenergetic function in a rat model of alcohol-induced fatty liver disease. Redox Biol. 9, 188-197. doi: 10.1016/j.redox.2016.08.005

Kirpich, I., Ghare, S., Zhang, J., Gobejishvili, L., Kharebava, G., Barve, S. J., et al. (2012). Binge alcohol-induced microvesicular liver steatosis and injury are associated with down-regulation of hepatic hdac 1, 7, 9, 10, 11 and upregulation of hdac 3. Alcohol Clin. Exp. Res. 36, 1578-1586. doi: 10.1111/j.15300277.2012.01751.x

Klein, M. A., Liu, C., Kuznetsov, V. I., Feltenberger, J. B., Tang, W., and Denu, J. M. (2020). Mechanism of activation for the sirtuin 6 protein deacylase. J. Biol. Chem. 295, 1385-1399. doi: 10.1074/jbc.RA119.011285

Kouzarides, T. (2007). Chromatin modifications and their function. Cell 128, 693-705. doi: 10.1016/j.cell.2007.02.005

Krützfeldt, J., Rajewsky, N., Braich, R., Rajeev, K. G., Tuschl, T., Manoharan, M., et al. (2005). Silencing of micrornas in vivo with 'antagomirs'. Nature 438, 685-689. doi: 10.1038/nature04303

Lawrence, T. (2009). The nuclear factor nf-kappab pathway in inflammation. Cold Spring Harb. Perspect Biol. 1:a001651. doi: 10.1101/cshperspect.a001651

Lee, Y. J., and Shukla, S. D. (2007). Histone h3 phosphorylation at serine 10 and serine 28 is mediated by p38 mapk in rat hepatocytes exposed to ethanol and acetaldehyde. Eur. J. Pharmacol. 573, 29-38. doi: 10.1016/j.ejphar.2007. 06.049

Lefebvre, B., Ozato, K., and Lefebvre, P. (2002). Phosphorylation of histone h3 is functionally linked to retinoic acid receptor beta promoter activation. EMBO Rep. 3, 335-340. doi: 10.1093/embo-reports/kvf066

Lieber, C. S. (2002). S-adenosyl-1-methionine and alcoholic liver disease in animal models: implications for early intervention in human beings. Alcohol 27, 173-177. doi: 10.1016/s0741-8329(02)00230-6

Liu, H., Gong, M., French, B. A., Li, J., Tillman, B., and French, S. W. (2014). Mallory-denk body (mdb) formation modulates ufmylation expression epigenetically in alcoholic hepatitis (ah) and non-alcoholic steatohepatitis (nash). Exp. Mol. Pathol. 97, 477-483. doi: 10.1016/j.yexmp.2014.10.001

Liu, M., Wang, Z., Ren, M., Yang, X., Liu, B., Qi, H., et al. (2019). Sirt4 regulates pten stability through ide in response to cellular stresses. Faseb J. 33, 5535-5547. doi: 10.1096/fj.201801987R

European Association for the Study of Liver (2012). Easl clinical practical guidelines: management of alcoholic liver disease. J. Hepatol 57, 399-420. doi: 10.1016/j.jhep.2012.04.004

Lo, W. S., Trievel, R. C., Rojas, J. R., Duggan, L., Hsu, J. Y., Allis, C. D., et al. (2000). Phosphorylation of serine 10 in histone h3 is functionally linked in vitro and in vivo to gen5-mediated acetylation at lysine 14. Mol. Cell 5, 917-926. doi: 10.1016/s1097-2765(00)80257-9

Ma, Y., Chai, H., Ding, Q., Qian, Q., Yan, Z., Ding, B., et al. (2019). Hepatic sirt3 upregulation in response to chronic alcohol consumption contributes to alcoholic liver disease in mice. Front. Physiol. 10:1042. doi: 10.3389/fphys.2019. 01042

Mandrekar, P. (2011). Epigenetic regulation in alcoholic liver disease. World J. Gastroenterol. 17, 2456-2464. doi: 10.3748/wjg.v17.i20.2456

McDaniel, K., Herrera, L., Zhou, T., Francis, H., Han, Y., Levine, P., et al. (2014). The functional role of micrornas in alcoholic liver injury. J. Cell. Mol. Med. 18, 197-207. doi: 10.1111/jcmm. 12223 
Meroni, M., Longo, M., Rametta, R., and Dongiovanni, P. (2018). Genetic and epigenetic modifiers of alcoholic liver disease. Int. J. Mol. Sci. 19:3857. doi: 10.3390/ijms19123857

Min, Z., Gao, J., and Yu, Y. (2018). The roles of mitochondrial sirt4 in cellular metabolism. Front. Endocrinol. (Lausanne) 9:783. doi: 10.3389/fendo.2018. 00783

Moghe, A., Joshi-Barve, S., Ghare, S., Gobejishvili, L., Kirpich, I., McClain, C. J., et al. (2011). Histone modifications and alcohol-induced liver disease: are altered nutrients the missing link? World J. Gastroenterol. 17, 2465-2472. doi: 10.3748/wjg.v17.i20.2465

Momen-Heravi, F., Saha, B., Kodys, K., Catalano, D., Satishchandran, A., and Szabo, G. (2015). Increased number of circulating exosomes and their microRNA cargos are potential novel biomarkers in alcoholic hepatitis. $J$. Transl. Med. 12:261. doi: 10.1186/s12967-015-0623-9

Mohrin, M., Shin, J., Liu, Y., Brown, K., Luo, H., Xi, Y., et al. (2015). Stem cell aging. a mitochondrial upr-mediated metabolic checkpoint regulates hematopoietic stem cell aging. Science 347, 1374-1377. doi: 10.1126/science.aaa2361

Morera, L., Lübbert, M., and Jung, M. (2016). Targeting histone methyltransferases and demethylases in clinical trials for cancer therapy. Clin. Epigenetics 8:57. doi: 10.1186/s13148-016-0223-4

Nagy, L. E. (2015). The role of innate immunity in alcoholic liver disease. Alcohol Res. 37, 237-250.

Nathan, D., Ingvarsdottir, K., Sterner, D. E., Bylebyl, G. R., Dokmanovic, M., Dorsey, J. A., et al. (2006). Histone sumoylation is a negative regulator in saccharomyces cerevisiae and shows dynamic interplay with positive-acting histone modifications. Genes Dev. 20, 966-976. doi: 10.1101/gad.1404206

Nisticò, R., Ferraina, C., Marconi, V., Blandini, F., Negri, L., Egebjerg, J., et al. (2014). Age-related changes of protein sumoylation balance in the a $\beta p p \operatorname{tg} 2576$ mouse model of alzheimer's disease. Front. Pharmacol. 5:63. doi: 10.3389/fphar. 2014.00063

North, B. J., and Verdin, E. (2007). Interphase nucleo-cytoplasmic shuttling and localization of sirt2 during mitosis. PLoS One 2:e784. doi: 10.1371/journal.pone. 0000784

Onn, L., Portillo, M., Ilic, S., Cleitman, G., Stein, D., Kaluski, S., et al. (2020). Sirt6 is a DNA double-strand break sensor. Elife 9:e51636. doi: 10.7554/eLife.51636

Osna, N. A., Donohue, T. M., and Kharbanda, K. K. (2017). Alcoholic liver disease: pathogenesis and current management. Alcohol Res. 38, 147-161.

Pal-Bhadra, M., Bhadra, U., Jackson, D. E., Mamatha, L., Park, P. H., and Shukla, S. D. (2007). Distinct methylation patterns in histone h3 at lys-4 and lys-9 correlate with up- \& down-regulation of genes by ethanol in hepatocytes. Life Sci. 81, 979-987. doi: 10.1016/j.lfs.2007.07.030

Park, P. H., Miller, R., and Shukla, S. D. (2003). Acetylation of histone h3 at lysine 9 by ethanol in rat hepatocytes. Biochem. Biophys. Res. Commun. 306, 501-504. doi: 10.1016/s0006-291x(03)01040-4

Park, P. H., Lim, R. W., and Shukla, S. D. (2005). Involvement of histone acetyltransferase (hat) in ethanol-induced acetylation of histone h3 in hepatocytes: potential mechanism for gene expression. Am. J. Physiol. Gastrointest. Liver Physiol. 289, G1124-G1136. doi: 10.1152/ajpgi.00091.2005

Park, P. H., Lim, R. W., and Shukla, S. D. (2012). Gene-selective histone h3 acetylation in the absence of increase in global histone acetylation in liver of rats chronically fed alcohol. Alcohol Alcohol. 47, 233-239. doi: 10.1093/alcalc/ ags004

Parker, R., Kim, S. J., and Gao, B. (2018). Alcohol, adipose tissue and liver disease: mechanistic links and clinical considerations. Nat. Rev. Gastroenterol. Hepatol. 15, 50-59. doi: 10.1038/nrgastro.2017.116

Parker, R., Kim, S. J., Im, G. Y., Nahas, J., Dhesi, B., Vergis, N., et al. (2019). Obesity in acute alcoholic hepatitis increases morbidity and mortality. EBioMedicine 45, 511-518. doi: 10.1016/j.ebiom.2019.03.046

Pochareddy, S., and Edenberg, H. J. (2012). Chronic alcohol exposure alters gene expression in hepg2 cells. Alcohol Clin. Exp. Res. 36, 1021-1033. doi: 10.1111/j. 1530-0277.2011.01677.x

Polletta, L., Vernucci, E., Carnevale, I., Arcangeli, T., Rotili, D., Palmerio, S., et al. (2015). Sirt5 regulation of ammonia-induced autophagy and mitophagy. Autophagy 11, 253-270. doi: 10.1080/15548627.2015.1009778

Purushotham, A., Schug, T. T., Xu, Q., Surapureddi, S., Guo, X., and Li, X. (2009). Hepatocyte-specific deletion of sirt1 alters fatty acid metabolism and results in hepatic steatosis and inflammation. Cell Metab. 9, 327-338. doi: 10.1016/j.cmet. 2009.02.006

Rehm, J., Samokhvalov, A. V., and Shield, K. D. (2013). Global burden of alcoholic liver diseases. J. Hepatol. 59, 160-168. doi: 10.1016/j.jhep.2013. 03.007

Restrepo, R. J., Lim, R. W., Korthuis, R. J., and Shukla, S. D. (2017). Binge alcohol alters pnpla3 levels in liver through epigenetic mechanism involving histone h3 acetylation. Alcohol 60, 77-82. doi: 10.1016/j.alcohol.2017.01.009

Rubio, M. D., Wood, K., Haroutunian, V., and Meador-Woodruff, J. H. (2013). Dysfunction of the ubiquitin proteasome and ubiquitin-like systems in schizophrenia. Neuropsychopharmacology 38, 1910-1920. doi: 10.1038/npp. 2013.84

Schueller, F., Roy, S., Vucur, M., Trautwein, C., Luedde, T., and Roderburg, C. (2018). The role of mirnas in the pathophysiology of liver diseases and toxicity. Int. J. Mol. Sci. 19:261. doi: 10.3390/ijms19010261

Seitz, H. K., Bataller, R., Cortez-Pinto, H., Gao, B., Gual, A., Lackner, C., et al. (2018). Alcoholic liver disease. Nat. Rev. Dis. Primers 4:16. doi: 10.1038/s41572018-0014-7

Shen, H., French, B. A., Tillman, B. C., Li, J., and French, S. W. (2015). Increased DNA methylation in the livers of patients with alcoholic hepatitis. Exp. Mol. Pathol. 99, 326-329. doi: 10.1016/j.yexmp.2015.08.001

Shepard, B. D., Joseph, R. A., Kannarkat, G. T., Rutledge, T. M., Tuma, D. J., and Tuma, P. L. (2008). Alcohol-induced alterations in hepatic microtubule dynamics can be explained by impaired histone deacetylase 6 function. Hepatology 48, 1671-1679. doi: 10.1002/hep.22481

Shimazu, T., Hirschey, M. D., Hua, L., Dittenhafer-Reed, K. E., Schwer, B., Lombard, D. B., et al. (2010). Sirt3 deacetylates mitochondrial 3-hydroxy3-methylglutaryl coa synthase 2 and regulates ketone body production. Cell Metab. 12, 654-661. doi: 10.1016/j.cmet.2010.11.003

Stickel, F., and Seitz, H. K. (2010). Alcoholic steatohepatitis. Best Pract. Res. Clin. Gastroenterol. 24, 683-693. doi: 10.1016/j.bpg.2010.07.003

Sun, R. P., Xi, Q. Y., Sun, J. J., Cheng, X., Zhu, Y. L., Ye, D. Z., et al. (2016). In low protein diets, microrna-19b regulates urea synthesis by targeting sirt5. Sci. Rep. 6:33291. doi: 10.1038/srep33291

Tang, Y., Zhang, L., Forsyth, C. B., Shaikh, M., Song, S., and Keshavarzian, A. (2015). The role of mir-212 and inos in alcohol-induced intestinal barrier dysfunction and steatohepatitis. Alcohol Clin. Exp. Res. 39, 1632-1641. doi: 10.1111/acer.12813

Teschke, R. (2018). Alcoholic steatohepatitis (ash) and alcoholic hepatitis (ah): cascade of events, clinical aspects, and pharmacotherapy options. Expert. Opin. Pharmacother. 19, 779-793. doi: 10.1080/14656566.2018.1465929

Thomson, S., Clayton, A. L., and Mahadevan, L. C. (2001). Independent dynamic regulation of histone phosphorylation and acetylation during immediateearly gene induction. Mol. Cell 8, 1231-1241. doi: 10.1016/s1097-2765(01)00 404-X

Tsai, Y. C., Greco, T. M., Boonmee, A., Miteva, Y., and Cristea, I. M. (2012). Functional proteomics establishes the interaction of sirt7 with chromatin remodeling complexes and expands its role in regulation of rna polymerase i transcription. Mol. Cell. Proteomics 11, 60-76. doi: 10.1074/mcp.A111.015156

Vaquero, A., Scher, M. B., Lee, D. H., Sutton, A., Cheng, H. L., Alt, F. W., et al. (2006). Sirt2 is a histone deacetylase with preference for histone h4 lys 16 during mitosis. Genes Dev. 20, 1256-1261. doi: 10.1101/gad.1412706

Vilà, L., Elias, I., Roca, C., Ribera, A., Ferré, T., Casellas, A., et al. (2014). Aav8-mediated sirtl gene transfer to the liver prevents high carbohydrate dietinduced nonalcoholic fatty liver disease. Mol. Ther. Methods Clin. Dev. 1:14039. doi: 10.1038/mtm.2014.39

Wang, C., Wan, X., Yu, T., Huang, Z., Shen, C., Qi, Q., et al. (2020). Acetylation stabilizes phosphoglycerate dehydrogenase by disrupting the interaction of e3 ligase rnf5 to promote breast tumorigenesis. Cell Rep. 32:108021. doi: 10.1016/ j.celrep.2020.108021

Wei, Y., and Xu, X. (2016). Ufmylation: a unique \& fashionable modification for life. Genomics Proteomics Bioinformatics 14, 140-146. doi: 10.1016/j.gpb.2016. 04.001

Yamamoto, Y., Verma, U. N., Prajapati, S., Kwak, Y. T., and Gaynor, R. B. (2003). Histone h3 phosphorylation by ikk-alpha is critical for cytokine-induced gene expression. Nature 423, 655-659. doi: 10.1038/nature01576 
Yeung, F., Hoberg, J. E., Ramsey, C. S., Keller, M. D., Jones, D. R., Frye, R. A., et al. (2004). Modulation of nf-kappab-dependent transcription and cell survival by the sirt1 deacetylase. Embo J. 23, 2369-2380. doi: 10.1038/sj.emboj.760 0244

Yin, H., Hu, M., Zhang, R., Shen, Z., Flatow, L., and You, M. (2012). Microrna-217 promotes ethanol-induced fat accumulation in hepatocytes by down-regulating sirt1. J. Biol. Chem. 287, 9817-9826. doi: 10.1074/jbc.M111.333534

Yin, H., Hu, M., Liang, X., Ajmo, J. M., Li, X., Bataller, R., et al. (2014). Deletion of sirt 1 from hepatocytes in mice disrupts lipin-1 signaling and aggravates alcoholic fatty liver. Gastroenterology 146, 801-811. doi: 10.1053/j.gastro.2013. 11.008

You, M., Cao, Q., Liang, X., Ajmo, J. M., and Ness, G. C. (2008). Mammalian sirtuin 1 is involved in the protective action of dietary saturated fat against alcoholic fatty liver in mice. J. Nutr. 138, 497-501. doi: 10.1093/jn/138.3.497

Zeybel, M., Hardy, T., Robinson, S. M., Fox, C., Anstee, Q. M., Ness, T., et al. (2015). Differential DNA methylation of genes involved in fibrosis progression in nonalcoholic fatty liver disease and alcoholic liver disease. Clin. Epigenetics 7:25. doi: 10.1186/s13148-015-0056-6

Zhang, J., Ren, D., Fedorova, J., He, Z., and Li, J. (2020). Sirt1/sirt3 modulates redox homeostasis during ischemia/reperfusion in the aging heart. Antioxidants (Basel) 9:858. doi: 10.3390/antiox9090858

Zhang, T., Yang, Z., Kusumanchi, P., Han, S., and Liangpunsakul, S. (2020). Critical role of microrna-21 in the pathogenesis of liver diseases. Front Med. (Lausanne) 7:7. doi: 10.3389/fmed.2020.00007
Zhang, X., Li, B., Rezaeian, A. H., Xu, X., Chou, P. C., Jin, G., et al. (2017). H3 ubiquitination by nedd4 regulates h3 acetylation and tumorigenesis. Nat. Commun. 8:14799. doi: 10.1038/ncomms14799

Zhang, Y. (2003). Transcriptional regulation by histone ubiquitination and deubiquitination. Genes Dev. 17, 2733-2740. doi: 10.1101/gad.1156403

Zhang, Y., Bharathi, S. S., Rardin, M. J., Lu, J., Maringer, K. V., Sims-Lucas, S., et al. (2017). Lysine desuccinylase sirt5 binds to cardiolipin and regulates the electron transport chain. J. Biol. Chem. 292, 10239-10249. doi: 10.1074/jbc. M117.785022

Zhong, X., Huang, M., Kim, H. G., Zhang, Y., Chowdhury, K., Cai, W., et al. (2020). Sirt6 protects against liver fibrosis by deacetylation and suppression of smad3 in hepatic stellate cells. Cell Mol. Gastroenterol. Hepatol. 10, 341-364. doi: 10.1016/j.jcmgh.2020.04.005

Conflict of Interest: The authors declare that the research was conducted in the absence of any commercial or financial relationships that could be construed as a potential conflict of interest.

Copyright (c) $2021 \mathrm{Kim}$, Cho, Kim and Kim. This is an open-access article distributed under the terms of the Creative Commons Attribution License (CC BY). The use, distribution or reproduction in other forums is permitted, provided the original author(s) and the copyright owner(s) are credited and that the original publication in this journal is cited, in accordance with accepted academic practice. No use, distribution or reproduction is permitted which does not comply with these terms. 